\title{
Endocrine gland-derived vascular endothelial growth factor modulates proliferation, apoptosis and migration in pancreatic cancer cells
}

\author{
LINAN REN, XIAOZHONG GUO, XIAODONG SHAO, HONGYU LI and HUI YAO
}

\author{
Department of Gastroenterology, Shenyang General Hospital of PLA, Shenyang, Liaoning 110840, P.R. China
}

Received January 15, 2014; Accepted September 18, 2014

DOI: $10.3892 / \mathrm{mmr} .2015 .3340$

\begin{abstract}
Endocrine gland-derived vascular endothelial growth factor (EG-VEGF) is a newly cloned factor that selectively acts on the endothelium of endocrine gland cells. EG-VEGF was previously identified as an important cytokine, involved in the modulation of apoptosis in pancreatic cancer cell lines. The present study examined the effects of EG-VEGF proliferation and migration, in pancreatic cancer cells. To determine the potential for EG-VEGF as a therapeutic target for pancreatic cancer, the expression of EG-VEGF were measured in pancreatic cancer tissue, and the association between its expression and the clinicopathological characteristics of the pancreatic cancer patients was determined. The results of the present study suggest that EG-VEGF may act as a novel tumor gene in pancreatic cancer. EG-VEGF was rarely expressed in the normal pancreatic tissue, but was highly expressed in the pancreatic cancer tissue. These data suggest that EG-VEGF may be a cancer-specific, and possibly tissue-specific, survival factor in the pancreas. In the Mia PaCa-2 pancreatic cancer cell line, EG-VEGF was shown to promote proliferation and cellular invasion, and modulate the phosphorylation of mitogen-activated protein kinase, a modulator for the malignant phenotype.
\end{abstract}

\section{Introduction}

Pancreatic cancer constitutes $\sim 3 \%$ of all diagnosed cancers; however, it is the third and fourth most common cause of cancer-associated mortality in females and males, respectively. Pancreatic cancer ranks 10th in the number of new cases, and 4 th in cancer-associated mortality in the US (1). There is no specific treatment for pancreatic cancer; therefore, patients suffering from pancreatic cancer have a very poor prognosis, with a 5-year relative survival rate of $<5 \%$ (2). Currently,

Correspondence to: Dr Xiaozhong Guo, Department of Gastroenterology, Shenyang General Hospital of PLA, 83 Wenhua Road, Shenyang, Liaoning 110840, P.R. China

E-mail: jie.deng@hotmail.com

Key words: endocrine gland-derived vascular endothelial growth factor, pancreatic cancer, proliferation, apoptosis there are no successful treatments for this cancer; therefore, it is crucial for doctors and scientists to identify methods to improve the diagnosis and treatment of pancreatic cancer.

Endocrine gland-derived vascular endothelial growth factor (EG-VEGF), was first cloned by LeCourter et al (3) in 2001. EG-VEGF selectively acts on the endothelium of endocrine gland cells. The coding region of EG-VEGF encodes 305 amino acids, and has a molecular weight of $8.6 \mathrm{kDa}$. The mature protein has been predicted to consist of 86 amino acids, containing 10 cysteine residues. EG-VEGF has high homology $(80 \%)$ with a nontoxic protein purified from the venom of the black mamba snake, but has low homology with VEGF (4). Human EG-VEGF has been shown to exert its functions through two $\mathrm{G}$ protein-coupled receptors (GPCRs). The expression of EG-VEGF is induced by hypoxia, and the protein is expressed in various endocrine tissues, including the testis, adrenal gland, ovary and placenta. The effects of EG-VEGF appear to be restricted to endothelial cells derived from these endocrine tissues (3). In pancreatic cancer cells, EG-VEGF was previously shown to protect the cells from apoptosis, through the upregulation of the myeloid cell leukemia-1 protein (5), but no disruption was observed in the function of EG-VEGF in pancreatic cancer cells.

To investigate the effects on EG-VEGF, at the molecular level in pancreatic cancer, the expression of EG-VEGF was determined in human pancreatic cancer tissue. Furthermore, the association between EG-VEGF expression and clinicopathological characteristics was assessed, and the in vitro activity of EG-VEGF was observed in pancreatic cancer cells.

\section{Materials and methods}

Cell lines. The present study used the Mia PaCa-2 human pancreatic cancer cell line, which were provided by Dr Friess (University of Heidelberg, Heidelberg, Germany) and originally purchased from the American Type Culture Collection (ATTC CRL-1420; Manassas, VA, USA). The cells were cultured in Dulbecco's modified Eagle's medium (DMEM), supplemented with $10 \%$ fetal calf serum, $100 \mathrm{U} / \mathrm{ml}$ penicillin and $100 \mathrm{mg} / \mathrm{ml}$ streptomycin, and maintained at $37^{\circ} \mathrm{C}$ in a humidified atmosphere containing $5 \% \mathrm{CO}_{2}$.

Tissue chip. A tissue chip was constructed by Cybrdi (Potomac, MD, USA), which included 60 pancreatic cancer 
tissue samples and 10 normal pancreatic tissue samples. The majority of the pancreatic cancer patients were male (65\%), with 39 samples from males and 21 from females. The age of the patients with pancreatic carcinoma ranged between 34 and 86 years, with a median age of 65 years. A histopathological diagnosis was made by two experienced pathologists. The tumors were staged according to the International Union against Cancer's Tumor-Node-Metastasis classification, and then histologically subtyped and graded according to the World Health Organization guidelines (6).

Immunohistochemistry. Immunostaining for EG-VEGF was performed manually at room temperature, using the Ultra Sensitive Solid Phase Immunohistochemistry kit (Maixin Biotech, Fuzhou, China). Phosphate-buffered saline (PBS) was used as a negative control. A tumor epithelial cell proportion score was determined and a staining intensity score was calculated for each slide. Staining was assessed in numerous areas on the slide, and given $\mathrm{a}+$ or - score and the average score was then determined for each slide. The slides were microwaved in EDTA buffer ( $\mathrm{pH} \mathrm{8.0)}$ for $60 \mathrm{~min}$, for antigen retrieval. The slides were then rinsed with water, and $3 \%$ hydrogen peroxide was applied to the slides for $4 \mathrm{~min}$ at room temperature. A further rinse with Tris buffer was performed, prior to incubation of the slides with EG-VEGF antibody (1:100 dilution), or PBS (control) overnight, at $4^{\circ} \mathrm{C}$. The slides were rinsed twice with Tris buffer, and then incubated with Biotin (Ventana, Tucson, AZ, USA) for $10 \mathrm{~min}$, rinsed again as before, and incubated with streptavidin for 8 min. Following a final rinse with Tris buffer, chromogen (dimethylaminoazobenzene; Ventana) was applied for $8 \mathrm{~min}$, followed by a copper solution for $4 \mathrm{~min}$. Counterstaining was performed using a commercially prepared hematoxylin for 4 min. Post-counterstaining was performed with a bluing solution (Beijing Biosynthesis Biotechnology Co., Ltd, Beijing, China), and the slides were dehydrated and coverslipped with Permount (Beijing Biosynthesis Biotechnology Co., Ltd). A BenchMark XT (Ventana) was used to measure the staining. Two independent pathologists viewed and interpreted the stained slides without knowledge of the patient outcome. The intensity of immunostaining for EG-VEGF was visually scored, and stratified into four groups: No tumor cells $(-),<10 \%$ tumor cells $( \pm), 10-50 \%$ tumor cells $(+)$, or $>50 \%$ tumor cells $(++)$. Any cytoplasmic staining with EG-VEGF was considered to indicate positive expression. Staining in the basal epithelial cells of the normal pancreatic cancer epithelium served as an internal control.

Cell proliferation assay. The anti-proliferative effects of EG-VEGF on the Mia PaCa-2 cells were determined using the MTT dye uptake method. Briefly, the cells $\left(2.5 \times 10^{3} / \mathrm{ml}\right)$, with or without EG-VEGF (100 $\mathrm{ng} / \mathrm{ml})$, were incubated in triplicate in a 96 -well plate. The plates were then cultured for $0,24,48,72$ or $96 \mathrm{~h}$. At each time point, $5 \mathrm{mg} / \mathrm{ml}$ MTT dye was added to each well. Following a $4 \mathrm{~h}$ incubation, the formed crystals were dissolved in $0.04 \mathrm{~m} \mathrm{HCl}$, in isopropanol. Absorbance was measured at $490 \mathrm{~nm}$ using a microplate reader (Bio-Rad Laboratories, Hercules, CA, USA). The cell proliferation rates of the EG-VEGF-treated and untreated cells were compared.
Apoptosis assay. Apoptosis was measured using an Annexin-V/Fluorescein isothiocyanate (FITC) Apoptosis Detection kit (BD Pharmingen, San Diego, CA, USA) and a 4,6-diamidino-2-phenylindole staining kit. A total of $\sim 0.3 \times 10^{6}$ cells were seeded in each well of a six-well plate. The cells were grown for $24 \mathrm{~h}$ in DMEM, without fetal bovine serum (FBS), with or without EG-VEGF (100 ng/ml). Both adherent and floating cells were collected, washed twice with cold PBS, and resuspended in cold annexin-V binding buffer, at a concentration of $1 \times 10^{7}$ cells $/ \mathrm{ml}$. Propidium iodide (PI) staining was performed in order to identify the dead cells. The cells were incubated at $37^{\circ} \mathrm{C}$ for $24 \mathrm{~h}$. Following further washing, the pellets were resuspended in $100 \mu \mathrm{l}$ binding buffer, containing Annexin V-FITC. The cells were incubated in the dark at room temperature for $15 \mathrm{~min}$, washed once with Annexin binding buffer, and stained with $10 \mu \mathrm{l} \mathrm{PI}$, on ice, for $30 \mathrm{~min}$. The cell pellet was analyzed using a FACSCalibur flow cytometer (BD Biosciences, Franklin Lakes, NJ, USA). The untreated cells were stained using the same protocol, and served as the controls.

\section{Assessment of EG-VEGF migration}

Wound healing assay. A wound healing assay was performed to determine the effects of EG-VEGF on the motility of the Mia PaCa-2 cells $(n=3)$. The cells were seeded in complete medium (DMEM, supplemented with 5\% FBS), at a density of $4 \times 10^{5}$ cells/well in six-well plates. Once the cells had reached confluency, the complete medium was replaced with serum-free medium, and the cells were scratched using a sterile tip, in order to create an artificial wound. The cells were allowed to heal for the next $24 \mathrm{~h}$, with or without EG-VEGF treatment $(100 \mathrm{ng} / \mathrm{ml})$. Images were captured at regular time intervals ( 0 and $24 \mathrm{~h}$ ) using an IX70-CoolSNAP microscope-camera combination (Olympus Corp., Tokyo, Japan). The size of the wound was measured from three separate experiments. The results are presented as a percentage of the wound closure, $24 \mathrm{~h}$ following the treatment.

Cell migration assay. The rates of migration were evaluated using a Matrigel ${ }^{\circledR}$-based assay, as previously described. Briefly, the cells were plated in triplicate at $2.5 \times 10^{4}$ cells $/$ well in serum-free medium on $8 \mu \mathrm{m}$ pore polycarbonate membranes of Transwell chambers, precoated with Matrigel ${ }^{\circledR}$ (BD Biosciences). The lower Transwell chambers contained EG-VEGF (100 ng/ml), without serum, as a chemoattractant. Matrigel-coated filters were placed between the upper and lower compartments. The cells were suspended in DMEM at a concentration of $2.5 \times 10^{4}$ cells $/ \mathrm{ml}$ and then loaded onto the upper chambers. The cells that had migrated through the Matrigel-coated filters were recovered from the lower compartments following a $24 \mathrm{~h}$ incubation, and counted. To determine the number of cells migrating (uncoated membrane), or invading (extracellular matrix-coated membrane), through the membrane, the membranes were fixed and stained with $0.5 \%$ crystal violet solution. Following a wash with water, the non-migrating or non-invading cells were removed by wiping the top of the membrane with a cotton-wool tip. The cells which had migrated or invaded through the membrane were manually counted, using magnified (x200) digital pictures of the insert/membranes (five fields for each membrane). The 
experiment was performed at least twice, with each sample being run in triplicate. An invasion index was calculated as the number of cells invading through the membrane in the treated cell group, divided by the number of cells migrating through the membrane in the control group. The migration of the cells was calculated from the ratio of the number of cells recovered from the lower compartment : the total number of cells initially loaded into the upper compartment.

Western blot analysis. In order to investigate whether the MAPK pathway was activated in response to EG-VEGF, Mia $\mathrm{PaCa} 2$ cells were: 1) Incubated with various concentrations of $\operatorname{EGVEGF}(0,50,100$ and $200 \mathrm{ng} / \mathrm{ml})$ for $30 \mathrm{~min}$; or 2) pretreated with $200 \mathrm{ng} / \mathrm{ml}$ Pertussin toxin (PTX) and $50 \mathrm{ng} / \mathrm{ml}$ PD98059, an MEK1 (MAPK2)-specific-inhibitor, for $1 \mathrm{~h}$ followed by exposure to $100 \mathrm{ng} / \mathrm{ml} \mathrm{EG-VEGF}$ for $30 \mathrm{~min}$ at $37^{\circ} \mathrm{C}$ with $5 \%$ $\mathrm{CO}_{2}$. The cells were then centrifuged, washed with cold PBS, and lysed on ice for $30 \mathrm{~min}$, using a lysis buffer containing protease and phosphatase inhibitors. The concentration of the protein samples were determined using a Bio-Rad protein assay (Bio-Rad Laboratories). Total protein was separated by electrophoresis on a $12 \%$ SDS-polyacrylamide gel, and transferred to a polyvinylidene fluoride membrane (Thermo Fisher Scientific, Inc., Rockford, IL, USA). The membranes were then blocked with 5\% non-fat milk and incubated with the following primary antibodies: Anti-EG-VEGF (1:200 dilution; BD Pharmingen), anti-MAPK (1:1,000 dilution; Dako, Glostrup, Denmark) or anti- $\beta$-actin (Santa Cruz Biotechnology, Inc., Dallas, TX, USA). Following an overnight incubation at $4^{\circ} \mathrm{C}$, the membranes were washed and incubated with horseradish peroxidase-conjugated secondary antibodies for $1 \mathrm{~h}$. The bands were visualized using an Enhanced Chemiluminescence substrate (Thermo Fisher Scientific, Inc.). The bands were quantified using Image $\mathrm{Lab}^{\mathrm{TM}} 4.1$ software (Bio-Rad Laboratories, Inc.) for densitometric analysis.

Statistics. Statistical comparisons of significance between the expression and clinicopathological features of the pancreatic cancer patients were evaluated using Cochran-Mantel-Haenszel Statistics or Fisher's Exact. Statistical analysis was performed using SAS version 9.0 software (SAS Institute Inc., Cary, NC, USA). The results are expressed as the means of the percentage \pm the standard deviation of the mean. Two-tailed student's t-tests were used when appropriate for statistical analysis. A $\mathrm{P}<0.05$ was considered to indicate a statistically significant difference.

\section{Results}

EG-VEGF expression in pancreatic cancer and normal pancreatic tissue. Positive staining for EG-VEGF expression was observed in the pancreatic islets of $8 / 10$ normal patients. However, only $1 / 10$ normal pancreatic tissue samples stained positively for EG-VEGF, whereas 57/60 of the tissue samples from the pancreatic cancer patients stained positively (Fig. 1).

The expression of EG-VEGF in pancreatic carcinomas had a linear correlation with the clinical stage of pancreatic cancer $(0.0299, \mathrm{P}<0.05$; Table $\mathrm{I})$. The expression of EG-VEGF was much higher in the pancreatic cancer tissue from patients in the later stages of cancer. The differences
Table I. Association between clinicopathological characteristics of the pancreatic cancer patients and endocrine gland-derived vascular endothelial growth factor (EG-VEGF) expression.

\begin{tabular}{lccc}
\hline Characteristic & $\begin{array}{c}\text { Patients } \\
(\mathrm{n})\end{array}$ & $\begin{array}{c}\text { EG-VEGF Positive } \\
(\%)\end{array}$ & P-value \\
\hline Age (years) & & & \\
$<50$ & 16 & 100.0 & $>0.05$ \\
$\geq 50$ & 44 & 93.1 & \\
Gender & & & $>0.05$ \\
Male & 39 & 94.9 & \\
Female & 21 & 95.2 & \\
Stage & & & \\
I & 25 & 92.0 & $<0.05$ \\
II & 26 & 96.2 & \\
III & 3 & 100.0 & \\
IV & 6 & 100.0 & \\
\hline
\end{tabular}

in the expression of EG-VEGF between the pancreatic tumor and normal pancreas tissue were also statistically significant $(4.167 \mathrm{E}-08, \mathrm{P}<0.05)$. Furthermore, the expression of EG-VEGF was detected in the islet cells of the normal pancreatic tissue.

EG-VEGF promotes Mia PaCa-2 cell proliferation and protects the cells from apoptosis. As compared with the untreated cells, the cells exposed to EG-VEGF had an increased rate of proliferation 48, 72 and $96 \mathrm{~h}$, following treatment $(\mathrm{P}<0.05$; Fig. 2A). The Mia $\mathrm{PaCa}-2$ cells were starved for $24 \mathrm{~h}$, and stained with Annexin-V/PI, the apoptotic cells were then identified using a fluorescence microscope. In the serum-deprived, untreated cells, $27.74 \%$ were positive for annexin-V/FITC staining; whereas among the cells cultured in the presence of $100 \mathrm{ng} / \mathrm{ml}$ EG-VEGF, only $13.21 \%$ cells were positive for Annexin-V/FITC staining (Fig. 2B).

The effects of EG-VEGF on Mia PaCa-2 migration. The effects of EG-VEGF on the migration of the Mia PaCa-2 cells were then determined. Images of the Mia PaCa-2 cells were captured 0 and $24 \mathrm{~h}$ following wound simulation by scraping the cells with a pipette tip, and incubation with or without $100 \mathrm{ng} / \mathrm{ml} \mathrm{EG-VEGF}$ (Fig. 2C). Following a $24 \mathrm{~h}$ incubation, the wound was almost completely healed in the EG-VEGF treated cells. The closure of the wound reached $79 \pm 5.43 \%$ in the EG-VEGF treated cells, as compared with $39 \pm 2.65 \%$ in the untreated controls (Fig. 2D).

The effects of EG-VEGF on Mia PaCa-2 invasion. The role of EG-VEGF in the invasiveness of human pancreatic cancer cells was determined using a Matrigel ${ }^{\circledR}$ migration assay. The cells treated with EG-VEGF, had a significantly higher number of cells migrating through the Matrigel, as compared with the control cells $(\mathrm{P}<0.05)$. In the presence of $0,50,100$, or $200 \mathrm{ng} / \mathrm{ml}$ EG-VEGF, the number of invading 


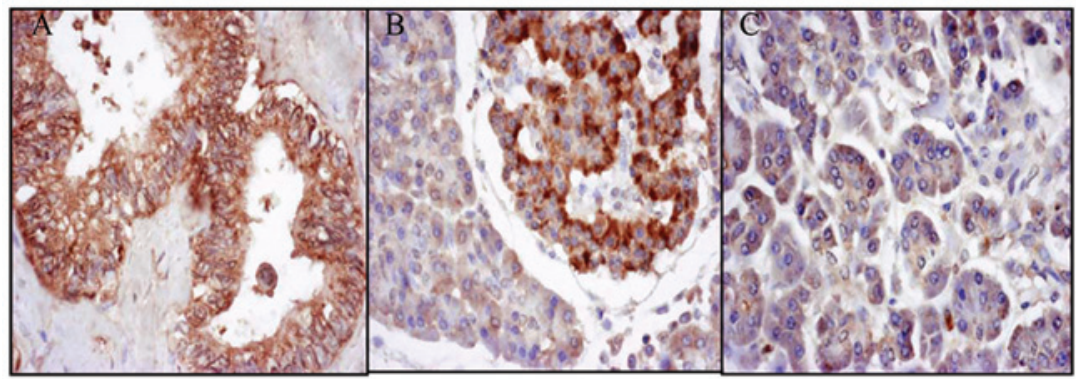

Figure 1. Immunolocalization of endocrine gland-derived vascular endothelial growth factor (EG-VEGF) in human pancreatic tumor and normal tissue samples. (A) Primary pancreatic cancer cells show a strong expression of EG-VEGF. (B) Positive EG-VEGF expression was also observed in the normal pancreatic islet cells. (C) Little or no EG-VEGF expression was detected in the normal pancreatic cells. Magnification, $\mathrm{x} 400$.

A

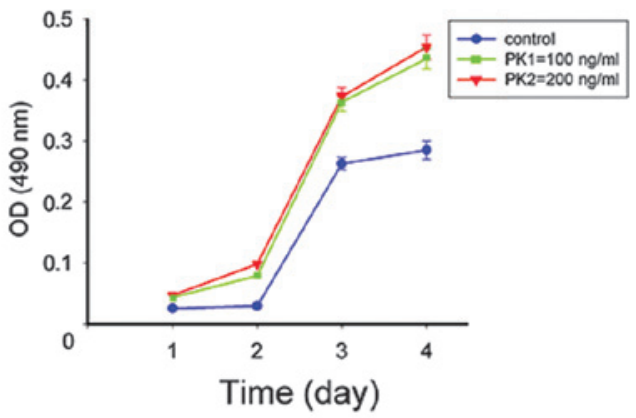

C

$\mathrm{Oh}$

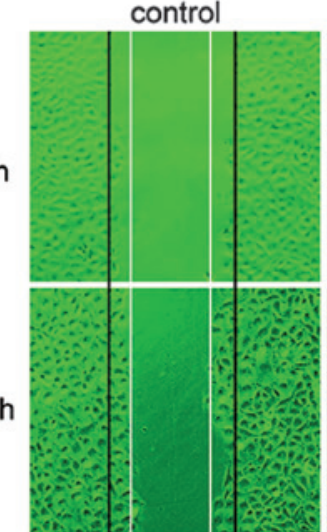

E

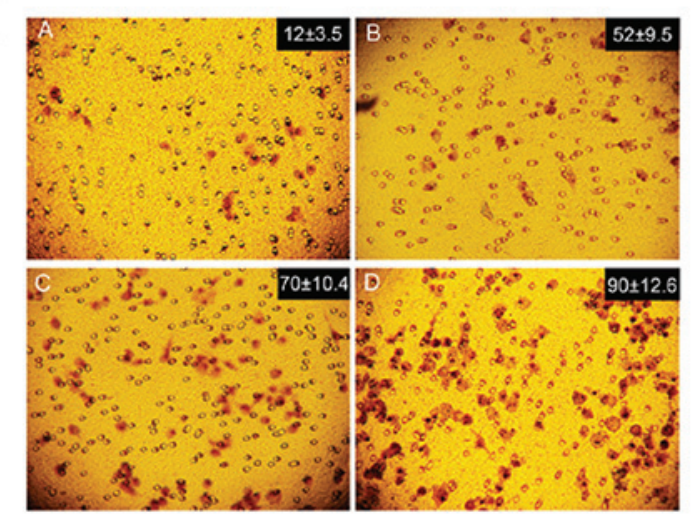

B
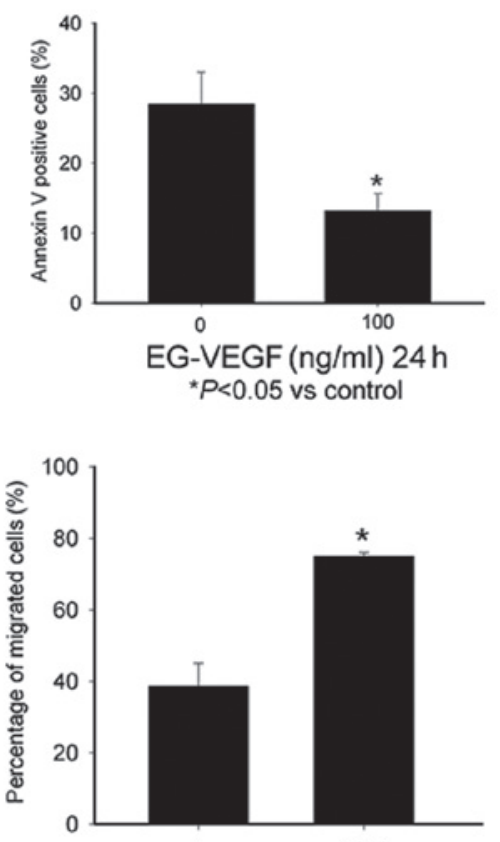

0

100

EG-VEGF (ng/ml)

${ }^{\star} P<0.05$ vs control

F

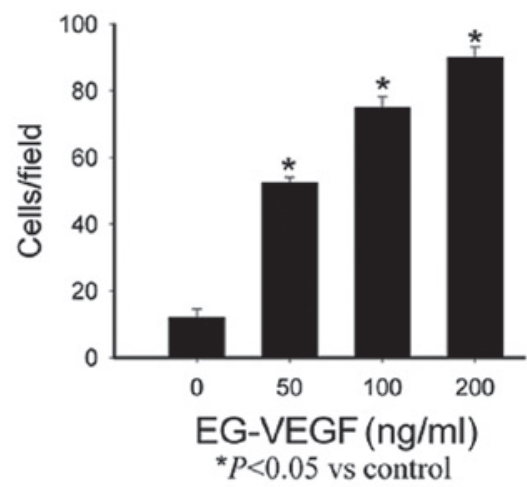

Figure 2. Endocrine gland-derived vascular endothelial growth factor (EG-VEGF) can modulate the malignant phenotype of the Mia PaCa-2 pancreatic cancer cells. (A) The Mia PaCa-2 cells were treated with EG-VEGF (100 or $200 \mathrm{ng} / \mathrm{ml}$ ), and cultured for 24, 48, 72, and 96 h. EG-VEGF treatment significantly promoted growth of the cells $(\mathrm{P}<0.05$, as compared with the control cells), but there was no marked difference between the $100 \mathrm{and} 200 \mathrm{ng} / \mathrm{ml}$ EG-VEGF groups. (B) Protective effects of EG-VEGF on the apoptosis of Mia PaCa-2 cells. The apoptotic rate of cells cultured in the absence of fetal bovine serum was $27.74 \%$, whereas the percentage of apoptosis of the cells cultured in the presence of EG-VEGF was significantly lower $(13.21 \%, \mathrm{P}<0.05)$. These data represent the average values derived from three independent experiments. ( $\mathrm{C}$ and $\mathrm{D})$ Effects of EG-VEGF on the migration of the Mia PaCa-2 cells. (C) Images of the Mia PaCa-2 cells $0 \mathrm{~h}$ and $24 \mathrm{~h}$ following the wounding, treated with $(100 \mathrm{ng} / \mathrm{ml})$ or without EG-VEGF. (D)The percentage of wound closure $24 \mathrm{~h}$ following treatment with EG-VEGF (100 ng/ml). (E) Images of the Mia PaCa-2 cells treated with EG-VEGF $(0,25,50,100 \mathrm{ng} / \mathrm{ml})$, following a migration assay. (F) The number of invasive cells was counted under a microscope. The data is expressed as the means \pm standard deviation of three independent experiments. "P<0.05, as compared with the control cells. OD, optical density; nm, nanometers. 
cells was $12 \pm 3.5,52 \pm 9.5,70 \pm 10.4$, and $90 \pm 12.6$ respectively (Fig. 2E and F).

EG-VEGF activates the MAPK pathway in Mia PaCa-2 cells. EG-VEGF induced the phosphorylation of p44/42 MAPK in the cultured Mia PaCa-2 pancreatic cancer cells. Numerous downstream signaling pathways were investigated that may potentially be associated with EG-VEGF signaling in Mia PaCa-2 cells. Following exposure of the cells to various concentrations of EG-VEGF for $30 \mathrm{~min}$, the cell lysates were separated by electrophoresis on an SDS-polyacrylamide gel, and transferred onto a polyvinylidene difluoride membrane. The membranes were then blotted with a phospho-p44/42 MAPK antibody (Fig. 3). A dose-dependent phosphorylation of p44/42 MAPK was observed, in response to EG-VEGF treatment. The effects of PTX were determined on EG-VEGF-induced MAPK activation. The MAPK activation was shown to be PTX-sensitive (Fig. 3). Pretreatment of the Mia PaCa-2 cells with $200 \mathrm{ng} / \mathrm{ml}$ PTX abolished EG-VEGF-induced p42/44 MAPK phosphorylation. Since PTX near completely abolished the EG-VEGF-induced phosphorylation of MAPK, it is likely that the EG-VEGF receptor is G protein coupled. To determine the specificity of the MAPK pathway being activated by EG-VEGF, the effects of the MEK1 (MAPK2)-spec ific-inhibitor PD98059 were examined on EG-VEGF-induced phosphorylation of p44/42 MAPK. Pretreatment of the Mia PaCa-2 cells with PD98059 blocked EG-VEGF-induced phosphorylation of p44/42 MAPK (Fig. 4).

\section{Discussion}

Patients suffering from pancreatic cancer have a very poor prognosis, with only 1-4\% of patients surviving for five years. Pancreatic ductal adenocarcinoma (PDAC), composing 70\% of all cases of pancreatic cancer, is not only the most common form, but also has the worst prognosis, of all of the pancreatic cancer subtypes (7). Currently, improvements in the treatment of pancreatic cancer patients are limited. Therefore, the identification of molecular markers that may aid the development of new therapeutic agents, is required.

There have been numerous reports on the role of EG-VEGF as a novel angiogenic factor expressed in malignant tumors (8-12), including colorectal cancer and metastatic renal cell carcinoma. Therefore, evaluation of the expression and action of EG-VEGF in pancreatic cancer may have important clinical applications. It was previously shown that EG-VEGF has a critical role in mediating anti-apoptotic effects in a pancreatic cancer cell line (5). The present study extended these findings, comparing the expression of EG-VEGF in pancreatic tumor and normal pancreatic tissues. The expression of EG-VEGF was significantly increased in the pancreatic cancer tissues, as compared with the normal pancreatic tissues. The expression of EG-VEGF was also shown to be much higher in the later stages of pancreatic cancer, and there was a linear correlation to this association. Therefore, it may be concluded that EG-VEGF has an important role in the development and progression of pancreatic neoplasms. Notably, the expression of EG-VEGF was also detected in the normal pancreatic islets. This phenomenon has also been observed in other studies $(13,14)$. The positive expression of the EG-VEGF in the
EG-VEGF (ng/ml) $30 \mathrm{~min}$

\begin{tabular}{|c|c|c|c|c|}
\hline & & & & \\
\hline & 0 & 50 & 100 & 200 \\
\hline p-42/44MAPK & - & & & \\
\hline
\end{tabular}

Figure 3. Endocrine gland-derived vascular endothelial growth factor (EG-VEGF) activated the mitogen-activated protein kinase (MAPK) pathway in Mia PaCa-2 pancreatic cancer cells. EG-VEGF induced p44/42 MAPK phosphorylation in a dose-dependent manner. Various concentrations of EG-VEGF $(0,50,100,200 \mathrm{ng} / \mathrm{ml})$ were added to Mia PaCa-2 cells and incubated for $30 \mathrm{~min}$. The cells were lysed, and the lysates were subjected to western blotting, using an anti-phospho-p44/42 MAPK antibody, and reprobed with anti-p44/42 MAPK to normalize the total proteins.

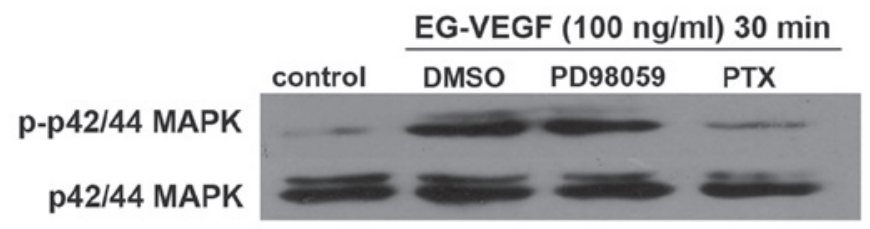

Figure 4. Endocrine gland-derived vascular endothelial growth factor (EG-VEGF)-induced activation of mitogen-activated protein kinase (MAPK) is Pertussin toxin (PTX)-sensitive. The Mia PaCa-2 pancreatic cancer cells were serum-starved overnight and pretreated with $200 \mathrm{ng} / \mathrm{ml}$ PTX and $50 \mathrm{ng} / \mathrm{ml}$ PD98059 for $1 \mathrm{~h}$. The cells were then incubated for $30 \mathrm{~min}$ at $37^{\circ} \mathrm{C}$ with $100 \mathrm{ng} / \mathrm{ml}$ EG-VEGF. The cell lysates were electrophoresed, transferred, and probed with phospho-p44/42 MAPK and p44/42 MAPK antibodies. DMSO, dimethyl sulfoxide.

normal pancreatic islets suggests a function for EG-VEGF in the mediation of endocrine function.

The malignant phenotype is an important biological phenomenon in cancer (15-18). Proliferation, migration, invasion and apoptosis are significant functions of cancer cells (19). It was previously demonstrated that EG-VEGF protects pancreatic cancer cells from apoptosis (4). In the present study EG-VEGF was shown to promote proliferation, apoptosis, migration and invasion in the Mia PaCa-2 pancreatic cell line. This function has also been reported in human neuroblastoma (20). The function of EG-VEGF in mediating the malignant phenotype may indicate that this cytokine is a crucial regulatory peptide in pancreatic cancer cells.

Further investigation is required to fully understand the regulatory mechanisms of EG-VEGF. The MAPK pathway is critical for cellular proliferation, migration and apoptosis (21), therefore the potential for EG-VEGF to stimulate p44/42 MAPK phosphorylation was also assessed in the present study. The results demonstrated that EG-VEGF was capable of inducing the activation of MAPK in pancreatic cancer cells, leading to proliferation, migration, and survival $(22,23)$.

The dose-dependent phosphorylation of MAPK p44/p42 is a sensitive indicator of EG-VEGF activity. The near complete inhibition of MAPK phosphorylation by pretreatment with the MEK-1 inhibitor PD-98059 indicated that the activation of MEK1/2 is necessary for EG-VEGF-induced MAPK p44/42 phosphorylation. These data suggest that the EG-VEGF receptor may be a GPCR mediated by MAPK activation. PTX has been shown to specifically modify the heterotrimeric 
G-protein Gai, blocking the signaling pathways of GPCRs which involve Gai. EG-VEGF-induced MAPK phosphorylation was also shown to be PTX-sensitive (24).

The results of the present study show a strong linear correlation between the expression of EG-VEGF in pancreatic tumors and the stage of pancreatic cancer. Furthermore, EG-VEGF modulated cellular proliferation, migration, and survival. In conclusion, the results suggest that EG-VEGF may be a potential candidate for gene therapy, or a target for other pancreatic cancer therapies.

\section{Acknowledgements}

The present study was supported in part by the Chinese National Natural Science Foundation (no. 81071982) and the Doctoral Research Found of Liaoning Province (no. 20111094).

\section{References}

1. Jemal A, Siegel R, Ward E, et al: Cancer statistics, 2006. CA Cancer J Clin 56: 106-130, 2006.

2. Wolfgang CL, Herman JM, Laheru DA, et al: Recent progress in pancreatic cancer. CA Cancer J Clin 63: 318-348, 2013.

3. LeCouter J, Kowalski J, Foster J, et al: Identification of an angiogenic mitogen selective for endocrine gland endothelium. Nature 412: 877-884, 2001.

4. Monnier J and Samson M: Cytokine properties of prokineticins. FEBS J 275: 4014-4021, 2008

5. Ren LN, Li QF, Xiao FJ, et al: Endocrine glands-derived vascular endothelial growth factor protects pancreatic cancer cells from apoptosis via upregulation of the myeloid cell leukemia-1 protein. Biochem Biophys Res Commun 386: 35-39, 2009.

6. Bosman FT (Eds.): WHO Classification of Tumours of the Digestive System. $4^{\text {th }}$ edition. IARC Press, Lyon, 2010.

7. Song JW and Lee JH: New morphological features for grading pancreatic ductal adenocarcinomas. Biomed Res Int 2013: $175271,2013$.

8. Nagano H, Goi T, Koneri K, et al: Endocrine gland-derived vascular endothelial growth factor (EG-VEGF) expression in colorectal cancer. J Surg Oncol 96: 605-610, 2007.

9. LeCouter J, Lin R, Tejada M, et al: The endocrine-gland-derived VEGF homologue Bv8 promotes angiogenesis in the testis: Localization of Bv8 receptors to endothelial cells. Proc Natl Acad Sci USA 100: 2685-2690, 2003.
10. Kisliouk T, Levy N, Hurwitz A and Meidan R: Presence and regulation of endocrine gland vascular endothelial growth factor/prokineticin-1 and its receptors in ovarian cells. J Clin Endocrinol Metab 88: 3700-3707, 2003.

11. Morales A, Vilchis F, Chávez B, et al: Expression and localization of endocrine gland-derived vascular endothelial growth factor (EG-VEGF) in human pancreas and pancreatic adenocarcinoma. J Steroid Biochem Mol Biol 107: 37-41, 2007.

12. Rini BI: Vascular endothelial growth factor-targeted therapy in metastatic renal cell carcinoma. Cancer 115: 2306-2312, 2009.

13. Jiang X, Abiatari I, Kong B, et al: Pancreatic islet and stellate cells are the main sources of endocrine gland-derived vascular endothelial growth factor/prokineticin-1 in pancreatic cancer. Pancreatology 9: 165-172, 2009.

14. Morales A, Morimoto S, Díaz L, Robles G and Díaz-Sánchez V: Endocrine gland-derived vascular endothelial growth factor in rat pancreas: genetic expression and testosterone regulation. J Endocrinol 197: 309-314, 2008

15. DingliD, ChalubFA, SantosFC, Van Segbroeck S andPacheco JM: Cancer phenotype as the outcome of an evolutionary game between normal and malignant cells. Br J Cancer 101: 1130-1136, 2009.

16. Stasinopoulos I, Mori N and Bhujwalla ZM: The malignant phenotype of breast cancer cells is reduced by COX-2 silencing. Neoplasia 10: 1163-1169, 2008.

17. Nielsen JD, Moeslund M, Wandall HH and Dabelsteen S: Influences of tumor stroma on the malignant phenotype. J Oral Pathol Med 37: 412-416, 2008.

18. Melnikova VO and Bar-Eli M: Transcriptional control of the melanoma malignant phenotype. Cancer Biol Ther 7: 997-1003, 2008.

19. Grzesiak JJ and Bouvet M: Divalent cations modulate the integrin-mediated malignant phenotype in pancreatic cancer cells. Cancer Sci 99: 1553-1563, 2008

20. Ngan ES, Sit FY, Lee K, et al: Implications of endocrine gland-derived vascular endothelial growth factor/prokineticin-1 signaling in human neuroblastoma progression. Clin Cancer Res 13: 868-875, 2007.

21. Koul HK, Pal M and Koul S: Role of p38 MAP kinase signal transduction in solid tumors. Genes Cancer 4: 342-359, 2013.

22. Arlt A, Müerköster SS and Schäfer H: Targeting apoptosis pathways in pancreatic cancer. Cancer Lett 332: 346-358, 2013.

23. Bayraktar S and Rocha-Lima CM: Advanced or metastatic pancreatic cancer: molecular targeted therapies. Mt Sinai J Med 77: 606-619, 2010.

24. Labasque M, Reiter E, Becamel C, et al: Physical interaction of calmodulin with the 5-hydroxytryptamine $2 \mathrm{C}$ receptor $\mathrm{C}$-terminus is essential for $\mathrm{G}$ protein-independent, arrestindependent receptor signaling. Mol Biol Cell 19: 4640-4650, 2008. 